\title{
Protein Binding to the Abscisic Acid-Responsive Element Is Independent of VIVIPAROUS1 in Vivo
}

\author{
Peter K. Busk ${ }^{1}$ and Montserrat Pagès ${ }^{2}$ \\ Departament de Genètica Molecular, Centre d'Investigació i Desenvolupament, Consell Superior d'Investigacions \\ Científiques, Jordi Girona 18-26, 08034 Barcelona, Spain
}

The plant hormone abscisic acid and the transcriptional activator VIVIPAROUS1 have a synergistic effect on transcription during embryo development. An abscisic acid-responsive element (ABRE) mediates induction by abscisic acid and VIVIPAROUS1, but the mechanism involved has not been determined. In this study, we explore the interaction between abscisic acid and VIVIPAROUS1 and its effect on the ABRE from the maize rab28 gene. In transient transformation experiments, abscisic acid stimulated transcription via several elements, whereas activation by VIVIPAROUS1 was mediated exclusively through the ABRE. In vivo footprinting showed only minor differences in binding to the ABRE between wild-type and VIVIPAROUS1-deficient embryos, suggesting that VIVIPAROUS1 stimulates transcription through the ABRE without major changes in protein-DNA interactions. A factor that bound to the ABRE in electrophoretic mobility shift assays was present at the same developmental stages as rab28 mRNA and had binding characteristics similar to those observed by in vivo footprinting. This suggests that the factor binds to the ABRE in the rab28 promoter in vivo. We discuss the constraints that our results put on the possible mechanism for action of VIVIPAROUS1 in vivo.

\section{INTRODUCTION}

The plant hormone abscisic acid (ABA) is a regulator of gene expression during embryo maturation (reviewed in Giraudat et al., 1994). ABA also plays a critical role in the response of vegetative tissue to stresses, such as drought, cold, and high salt concentrations (reviewed in Ingram and Bartels, 1996). Studies of the promoters of ABA-responsive genes have revealed an $A B A$-responsive element (ABRE) with the core sequence ACGT capable of mediating ABA-inducible transcription (Marcotte et al., 1989; Mundy et al., 1990). The ABRE is homologous to the G-box (Izawa et al., 1993), which is implicated in the response to various environmental stimuli, such as light (Giuliano et al., 1988), UV light (Weisshaar et al., 1991), and anaerobiosis (McKendree and Ferl, 1992). A variety of basic leucin zipper (bZIP) transcription factors binds to the element in vitro, but none has yet been shown unambiguously to mediate induction by $\mathrm{ABA}$ in vivo ( $\mathrm{Lu}$ et al., 1996).

The bases flanking the ACGT core of the ABRE play a crucial role in protein binding in vitro (lzawa et al., 1993) and affect the function in vivo (Salinas et al., 1992; Busk et al., 1997). However, the available data favor a model in which

\footnotetext{
tCurrent address: Afdeling for Plantebiokemi, Den Kongelige Veterinær- og Landbohøjskole, Thorvaldsensvej 40, 1870 Frederiksberg C, Denmark.

${ }^{2}$ To whom correspondence should be addressed. E-mail mptgmm@ cid.csic.es; fax 34-3-2045904.
}

the synergistic action of the ABRE together with other elements is necessary for the specificity of ABA-inducible promoters (Shen and Ho, 1995; Shen et al., 1996).

Genetic studies with maize have shown that mutants in the seed-specific developmental activator VIVIPAROUS1 (VP1) have reduced sensitivity to $A B A$ and do not express several ABA-inducible genes (reviewed in McCarty, 1995). In addition, ectopic expression of the ABA-insensitive VP1 homolog $A B \mid 3$ from Arabidopsis results in the induction of otherwise seed-specific genes in response to $A B A$ in vegetative tissues (Parcy et al., 1994).

Expression of the ABA-inducible genes $E m$ and rab28 in the maize embryo is controlled by VP1 (McCarty et al., 1991; Pla et al., 1991). However, VP1 is present at earlier developmental stages than are rab28 and Em mRNA. Therefore, other developmental cues are necessary for the expression of these genes. Transcription of the rab28 and $E m$ genes is low or absent in VP1-deficient embryos but can be induced by high concentrations of exogenously applied ABA (Pla et al., 1991; Williamson and Scandalios, 1992). Promoter analysis has shown that there is a synergistic effect of ABA and VP1 on the ABREs of Em (Hattori et al., 1995; Vasil et al., 1995). This effect mimics the developmental regulation of $\mathrm{Em}$. The ABRE could also be important for the regulation of rab28, because a sequence of $134 \mathrm{bp}$ with a putative ABRE is sufficient for induction by ABA of the rab28 promoter (Pla et al., 1993). Therefore, the ABRE is central for control of 


\section{ABRE A GRA -156 GCGCTCGC GCCACGTGGG CATGCCGCC GCG}

\begin{tabular}{|cr} 
CE3 & ABRE B \\
\hline ACGCGCCTCCTC TTGCTCGTC TCCACGTCTC TCG & -93
\end{tabular}

Figure 1. The Putative ABA-Responsive Sequences in the Minimal rab28 Promoter Inducible by $\mathrm{ABA}$.

The putative elements are enclosed in boxes. Numbering is relative to the transcription start point.

gene expression in the embryo. An understanding of how VP1 and ABA stimulate transcription through the ABRE will give important information about the regulation of embryogenesis.

In vitro, VP1 stimulates binding of the bZIP transcription factor EmBP-1 to the ABRE, probably by a transient or unstable interaction with the DNA (Hill et al., 1996). However, VP1 enhances binding in vitro of different classes of plant and animal transcription factors to their target sites in an apparently unspecific manner. Therefore, it remains to be determined how VP1 stimulates transcription through the ABRE in vivo.

We have used the rab28 promoter as a model system to understand the stimulation of transcription by VP1 and ABA via the ABRE. VP1 and ABA induced transcription through the two ABREs of the rab28 promoter in transient transformation assays in the same way as has been shown for $E m$. In vivo footprinting in wild-type and VP1-deficient embryos showed that protein binding to the ABREs is independent of VP1. However, there was a minor enhancement of the footprint in the presence of VP1. These results suggest that VP1 activates transcription via the ABRE, mainly by a mechanism that does not involve changes in protein-DNA interactions. The activity of an ABRE binding factor (ABF) from nuclear extracts correlated with the developmental expression of rab28. This factor could be binding to the ABREs of rab28 in vivo and mediating induction by VP1 and ABA.

\section{RESULTS}

\section{VP1- and ABA-Inducible Elements in the rab28 Promoter}

The sequence from 60 to $193 \mathrm{bp}$ upstream of the transcription start of the rab28 gene is sufficient to confer inducibility by $A B A$ on a cauliflower mosaic virus $35 S-90$ to +35 promoter in rice protoplasts (Pla et al., 1993). Four putative $A B A$-inducible elements present in this sequence are depicted in Figure 1. ABRE A binds nuclear proteins from embryos and leaves and was postulated to be involved in the ABA response of rab28 (Pla et al., 1993). ABRE B is another putative ABRE in the rab28 promoter. In addition, there is a sequence with similarity (seven of 12 bases) to the GRA element from the maize rab17 promoter (Busk et al., 1997) and a sequence with 10 of 12 bases identical to the coupling element CE3 from the HVA1 promoter from barley (Shen et al., 1996).

To define the function of these elements, we performed transient transformation of maize embryonic callus with the rab28 promoter construct pMP130 (Pla et al., 1993). The inducibility by $A B A$ of the wild-type construct and mutants of each of the two ABREs (ABRE $A^{-}$and $A B R E B^{-}$), the double mutant (ABRE $\left.A^{-} B^{-}\right)$, and a GRA mutant (GRA-) and a CE3 mutant $\left(C E 3^{-}\right)$was tested, and the results are presented in Figure 2. In callus, the ABRE $A^{-}$construct conferred weak induction by $A B A$ compared with that of the wild type, showing that $A B R E A$ is functional. In contrast, the ABRE $B^{-}$ construct had almost the same inducibility as the wild type did. Disruption of both of the ABREs (ABRE $A^{-} B^{-}$) abolished the activity of the promoter. The $\mathrm{GRA}^{-}$and $\mathrm{CE} 3^{-}$constructs had lowered inducibility, but it was not as low as that of ABRE $A^{-}$.

The developmental activation of rab28 depends on VP1 but can be partially overcome by exogenous ABA (Pla et al., 1991). We wanted to test whether VP1 enhances transcription through the four elements described above. Cotransformation of a VP1 expression plasmid with the rab28 promoter stimulated transcription (Figure 2). In addition, as shown in Table 1, induction by ABA and VP1 was cooperative.

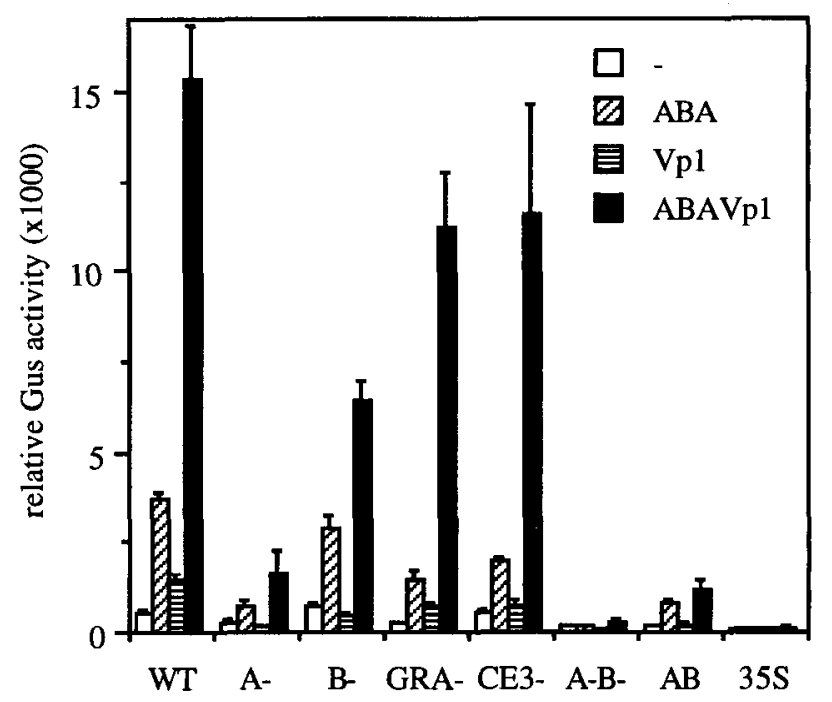

Figure 2. Activity of the rab28 Promoter Constructs in Transient Transformation of Maize Callus.

The values represent the mean of three experiments. Standard deviations are represented by error bars. Relative $\beta$-glucuronidase (GUS) activity was calculated as described in Methods. WT, wild type; $A-$, ABRE $A^{-} ; B-, A B R E B^{-} ; A-B-, A B R E A^{-} B^{-} ; A B$, the plasmid with $A B R E A$ and $A B R E B$ in front of a $35 S$ promoter. 
Table 1. Fold Induction by VP1 a

\begin{tabular}{lll}
\hline Construct $^{b}$ & $\begin{array}{l}\text { No } \\
\text { ABA }\end{array}$ & $\begin{array}{l}\text { ABA } \\
(100 \mu \mathrm{M})\end{array}$ \\
\hline WT & 2.6 & 4.1 \\
A $^{-}$ & 0.6 & 2.3 \\
B $^{-}$ & 0.6 & 2.3 \\
GRA $^{-}$ & 2.6 & 7.5 \\
CE3 $^{-}$ & 1.3 & 5.8 \\
\hline
\end{tabular}

a Calculated from the data shown in Figure 2 by dividing the activity of the construct when cotransformed with VP1 by the activity of the construct without VP1.

${ }^{b} W T$, wild type; $A^{-}, A_{B R E ~} A^{-} ; B^{-}, A B R E B^{-}$.

In the presence of VP1, the activities of ABRE $\mathrm{A}^{-}$and $\mathrm{ABRE} \mathrm{B}^{-}$were much lower than the activity of the wild-type promoter, suggesting that the ABREs mediated induction by VP1 (Figure 2). The two mutated constructs showed the same reduction in inducibility by VP1, suggesting that VP1 regulated transcription through $A B R E A$ and $A B R E B$ by using the same mechanism (Table 1). Curiously, VP1 repressed transcription of the $A B R E A^{-}$and $A B R E B^{-}$constructs in the absence of $A B A$. A construct with $A B R E A$ and $A B R E B$ in front of the 35S promoter demonstrated that the sequence of the ABREs was sufficient for induction by ABA and VP1, although the level of expression was much less than that of the wild-type construct (Figure 2). Without ABA, $\mathrm{CE}^{-}{ }^{-}$was less inducible by VP1 than was the wild type, but twice as inducible as ABRE $A^{-}$and ABRE $B^{-}$(Table 1). In the presence of $\mathrm{ABA}, \mathrm{GRA}^{-}$and $\mathrm{CE}^{-}$had the same fold of induction by VP1 as did the wild-type construct.

\section{In Vivo Footprinting of the rab28 Promoter in Wild-Type Embryos}

To investigate whether the results obtained by expression of the rab28 promoter in callus were relevant for the developmental activation of rab28, we performed in vivo footprinting of the rab28 promoter in embryos 28 days after pollination (DAP). In these embryos, the rab28 gene is developmentally expressed (Pla et al., 1991; Niogret et al., 1996).

Figures $3 \mathrm{~A}$ and $3 \mathrm{~B}$ show that there was a footprint on ABRE $A$, with protection of the guanines in the central sequence (CACGTG) and hypersensitivity of the flanking guanines. The protections were almost complete, showing that there was strong binding to the element. Qualitatively, the same footprint was observed on ABRE B; however, on this element, the central bases were less protected, indicating a lower affinity for the factor. The weaker footprint on ABRE B is in accordance with the activity of the ABRE $B^{-}$construct in transient transformation. There were no changes in the footprints on the two ABREs in excised embryos incubated for $24 \mathrm{hr}$ with ABA, whereas the footprint on ABRE B
A

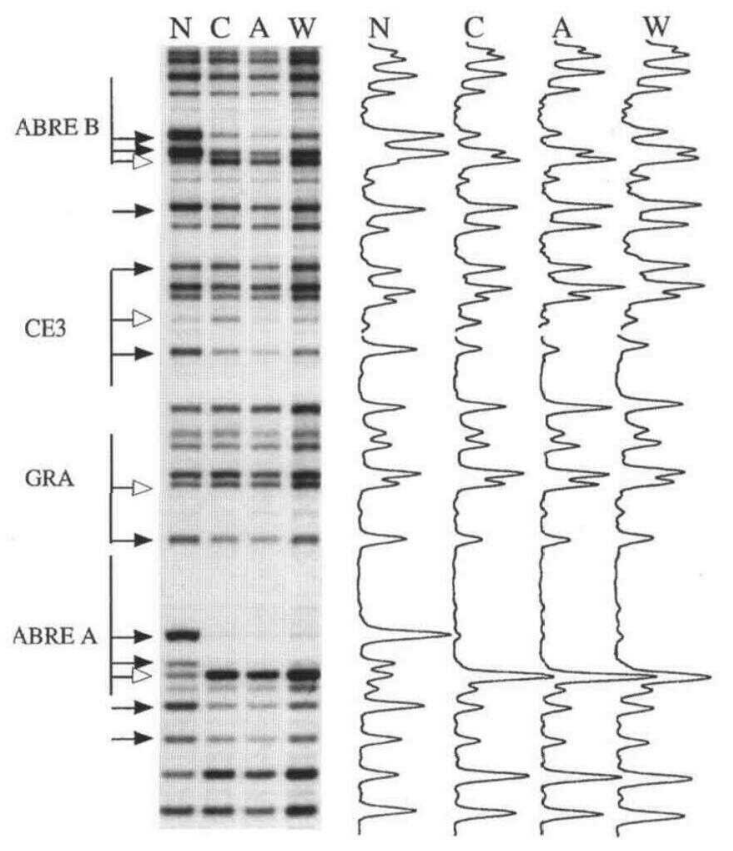

B

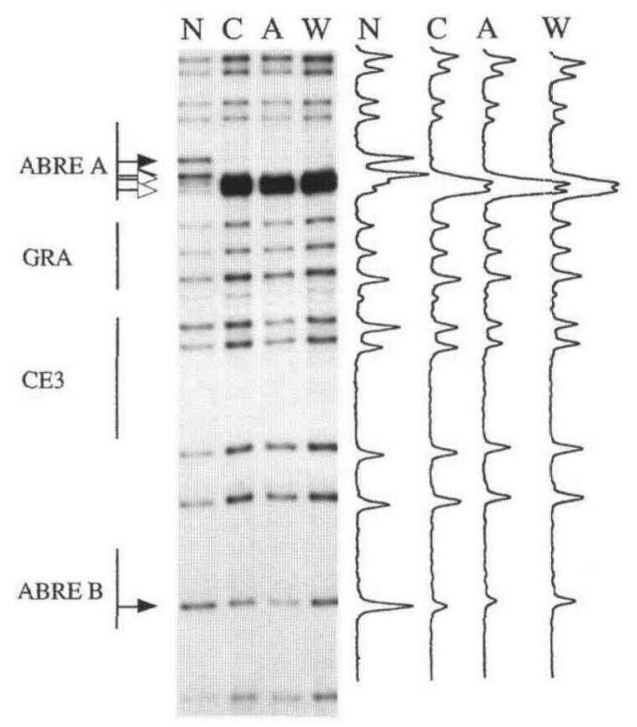

Figure 3. In Vivo Footprinting of the rab28 Promoter in 28-DAP Wild-Type Embryos.

In vitro-methylated DNA (lane N) compared with DNA from 28-DAP embryos treated with dimethylsulfate (DMS) within 15 min after excision from the cob (lane C) and DNA from embryos treated with DMS after $24 \mathrm{hr}$ of incubation with $100 \mu \mathrm{M}$ ABA (lane A) or water (lane W). (A) The nontranscribed strand from positions -91 to -155 .

(B) The transcribed strand from positions -93 to -156 .

The scanning profile of each lane is shown next to the sequence ladder. The extent of the elements mentioned in Figure 1 is indicated with bars. Filled arrows, reduced reactivity (protection) in vivo; open arrows, enhanced reactivity (hypersensitivity) in vivo. 
became weaker in embryos incubated without ABA (Figures $3 A$ and $3 B$ ).

We detected protein-DNA interactions on the two nonABRE elements. CE3 had two protections and a clear hypersensitivity, whereas the footprint on the GRA was weak (Figure $3 \mathrm{~A}$ ). Thus, the in vivo footprinting showed that all four elements studied by transient transformation bound proteins in vivo.

\section{In Vivo Footprinting in VP1-Deficient Embryos}

By in vivo footprinting of the vp1 mutant, we were able to determine whether VP1 induces transcription of rab28 by enhancing protein binding to the two ABREs in the promoter. Figures $4 A$ and $4 B$ show that there was a clear footprint on ABRE $A$ in VP1-deficient embryos. The footprint was slightly weaker than that in wild-type embryos, but the difference was at the limit of detection. This shows that VP1 had only a minor effect on protein binding to $A B R E A$ in vivo.

On ABRE B, the footprint in the vp1 mutant was weaker than that in the wild-type embryos (Figures $4 A$ and $4 B$ ). The difference between the wild type and vp1 was clearest on the protected residue $(-103)$ on the lower strand, which was more protected in the wild type than in the vp1 mutant (Figures $3 A$ and $4 A$ ).

In excised VP1-deficient embryos incubated with $A B A$, the protein-DNA interactions on ABRE $A$ and ABRE $B$ were weaker. Incubation of excised embryos in water clearly reduced the footprint on ABRE $A$, and the footprint on ABRE $B$ almost disappeared (Figures $4 A$ and $4 B$, lanes $A$ and $W$ ). This was different from the wild type and shows that VP1 deficiency leads to changes in the protein-DNA interactions on the ABREs in excised embryos.

Apart from the footprints, there are some differences in the sequence ladder of naked DNA (Figures $3 \mathrm{~A}$ and $4 \mathrm{~A}$, lanes $\mathrm{N}$ ) between the wild type and the vp1 mutant. These differences are found in the GRA and CE3 regions and are due to polymorphisms between the lines. The polymorphisms do not affect the regulation of the rab28 gene (Pla et al., 1991).

\section{A Developmentally Regulated ABF Binds to the Two ABREs in the rab28 Promoter with Different Affinity}

The different extent of protein binding to $A B R E A$ and $A B R E$ $B$ in vivo could be caused by different affinities for an $A B F$ or could be an effect of chromatin structure. Therefore, we performed electrophoretic mobility shift assays with nuclear extracts from embryos and oligonucleotides with the sequence of $A B R E A$ or ABRE $B$.

As shown in Figure $5 A$, a retarded complex was formed when nuclear extracts were incubated with ABRE $A$. The binding could be competed by 100 -fold excess of unlabeled ABRE $A$ but not with an ABRE $A^{-}$oligonucleotide, showing

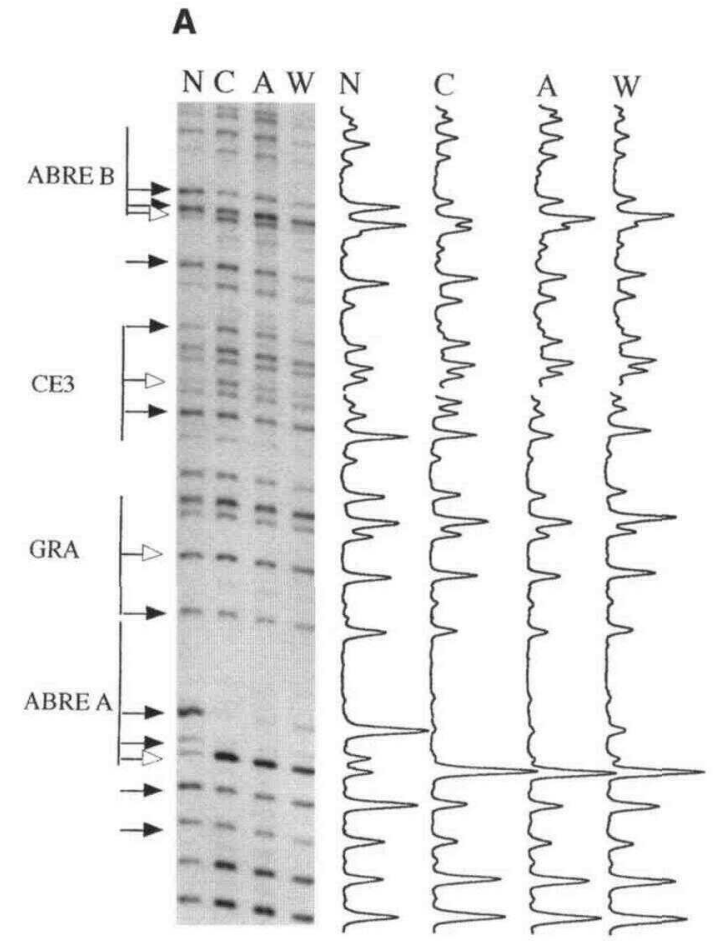

B

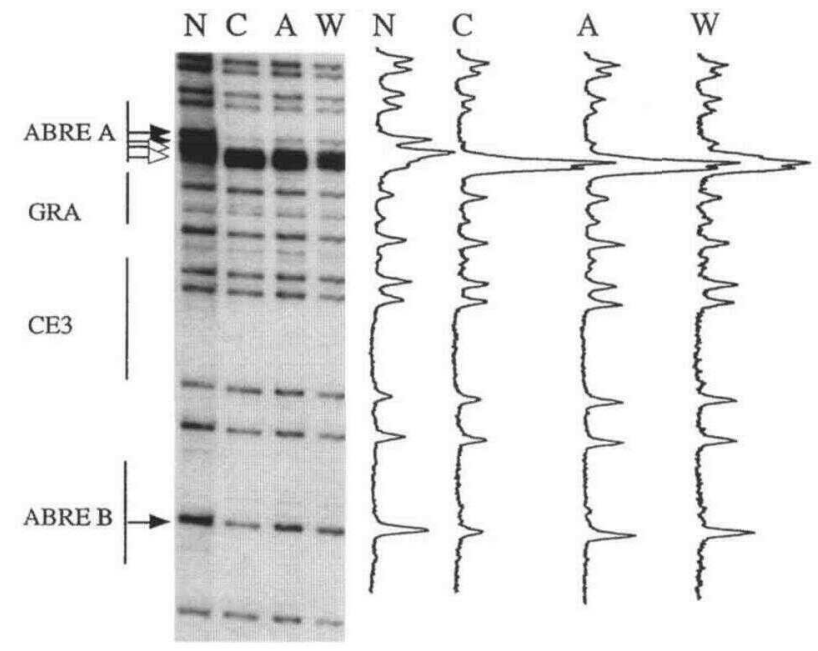

Figure 4. In Vivo Footprinting of the rab28 Promoter in 28-DAP vp1 Embryos.

(A) The nontranscribed strand from positions -91 to -155 .

(B) The transcribed strand from positions -93 to -156 . All symbols are as given in the legend to Figure 3. 
that binding was specific. Unlabeled ABRE $B$ competed less than did $A B R E A$, indicating that $A B R E B$ had a lower affinity for the ABF. When ABRE $B$ was used as a probe, the retarded complex was observed only upon long exposure (Figure 5B). Competition of this complex confirmed that an ABF from embryos bound better to ABRE $A$ than it did to ABRE B. Extracts from VP1-deficient embryos formed the same retarded complex (P.K. Busk and M. Pagès, unpublished data).

As shown in Figures $6 \mathrm{~A}$ and $6 \mathrm{~B}$, the $\mathrm{ABF}$ was present in nuclear extracts from 25- to 60-DAP embryos in which the rab28 gene was active. Only a weak band with the same mobility was observed in 14-DAP embryos in which rab28 was not transcribed (Figure 6B). However, extracts from 14DAP embryos formed complexes with different mobilities, suggesting that other factors with a similar specificity are present in young embryos. The activity of the ABF was highest in 25-DAP embryos and decreased when germination was induced by incubating the embryos in water. However, high activity of the ABF was observed in extracts from embryos incubated with ABA. These results show that there is a positive correlation between expression of rab28 and the activity of an ABF.

\section{DISCUSSION}

\section{Transactivation by VP1 through ABRE}

VP1 potentiates the response to $A B A$ in the maize embryo but is not an integrated part of the ABA signaling pathway (Hattori et al., 1992; Vasil et al., 1995). Embryogenesis and ABA induce the catalase1 gene independently of VP1, showing that VP1 does not activate all ABA-inducible genes (Williamson and Scandalios, 1992). Hill et al. (1996) proposed that VP1 only regulates embryo-specific genes. However, expression of rab28 is clearly lower in the vp1 mutant, although the gene is not embryo specific (Pla et al., 1991). In fact, both rab28 and the embryo-specific maize $\mathrm{Em}$ gene are dependent on VP1 for expression during normal seed development, but they can be induced by $A B A$ in excised VP1-deficient embryos (Pla et al., 1991; Williamson and Scandalios, 1992).

VP1 mediates activation through the ABREs of the promoters of the Em genes from cereals (Hattori et al., 1995; Vasil et al., 1995) in the same way as we show here for rab28. Stimulation of transcription by VP1 of the Em gene promoters depends on an acidic activation domain, indicating that VP1 contacts the transcriptional machinery (McCarty et al., 1991; Hattori et al., 1995). In addition, VP1 enhances binding of EmBP-1 to the ABRE in vitro (Hill et al., 1996). A domain of VP1 rich in basic amino acids is necessary for this function and for induction of $E m$ transcription. Here, we show that VP1 had little influence on protein binding to the ABREs of the rab28 promoter, as detected by in vivo footprinting.
A

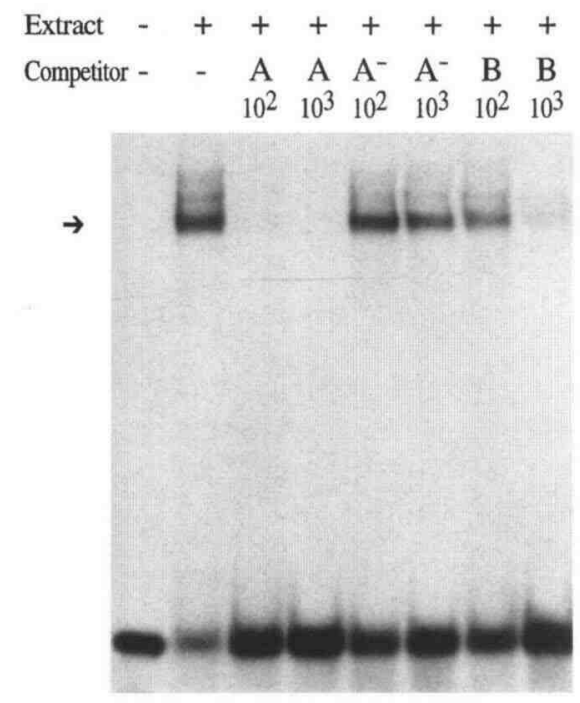

B

$\begin{array}{ccccccccc}\text { Extract - } & + & + & + & + & + & + & + \\ \text { Competitor - } & - & \text { B } & \text { B } & \mathrm{B}^{-} & \mathrm{B}^{-} & \mathrm{A} & \mathrm{A} \\ & & 10^{2} & 10^{3} & 10^{2} & 10^{3} & 10^{2} & 10^{3}\end{array}$

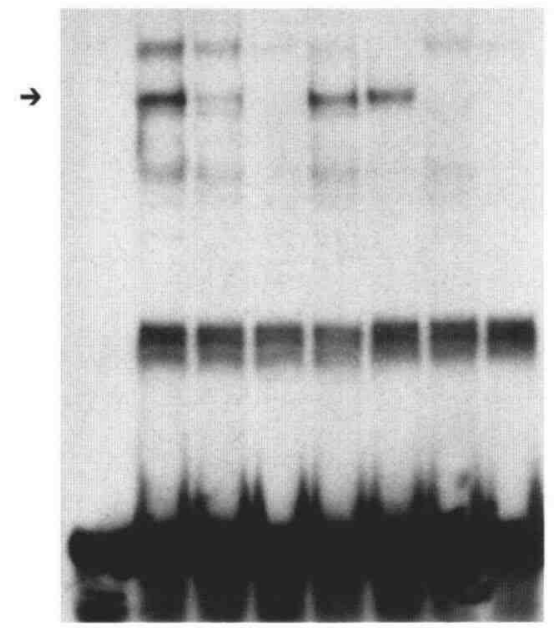

Figure 5. A Nuclear Protein Factor from Embryos Binds Specifically to the ABREs of the rab28 Promoter.

(A) Electrophoretic mobility shift assay with probe A (overnight exposure).

(B) Electrophoretic mobility shift assay with probe B (5-day exposure). Nuclear extracts $(45 \mu \mathrm{g})$ from 37-DAP embryos were preincubated with a 100- or 1000-time excess of unlabeled competitor, as shown above each lane. (-), probe without nuclear extract/competitor; (+), probe with the nuclear extract. The retarded complex mentioned in the text is indicated by an arrow. $A, A B R E A ; B, A B R E B ; A^{-}, A B R E$ $\mathrm{A}^{-} ; \mathrm{B}^{-}, \mathrm{ABRE} \mathrm{B}^{-}$. 
There was strong binding to the ABREs in the abscence of VP1. This observation excludes the possibility that VP1 is necessary for opening the chromatin structure of $A B A$ induced genes. Interestingly, EmBP-1 binds to the ABRE on nucleosomes and interacts with histone $\mathrm{H} 1$, suggesting that

A
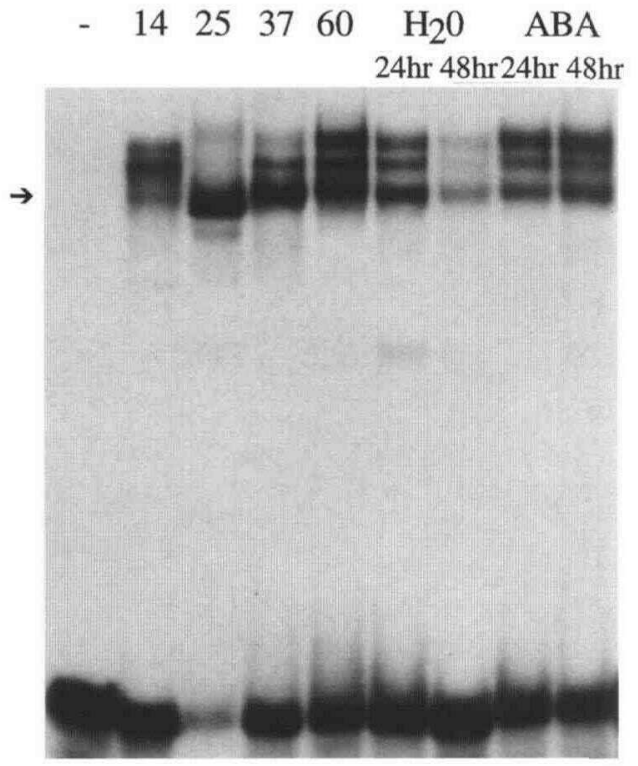

B

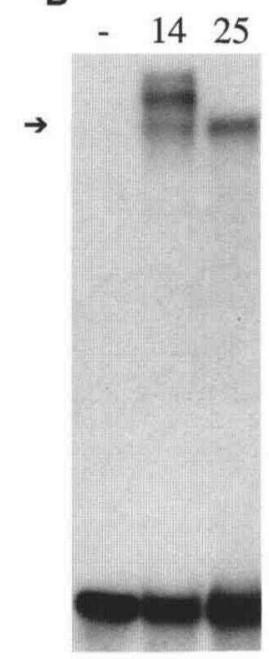

Figure 6. Activity of the ABF during Development.

(A) Electrophoretic mobility shift assay with probe $A$ and nuclear extracts from different developmental stages. The age of the embryos in days is indicated above each lane. $\mathrm{H}_{2} \mathrm{O}$, extract from embryos of mature seeds incubated in water for the time indicated; $A B A$, extract
EmBP-1 or related proteins could be responsible for providing and maintaining an open chromatin structure on ABREcontaining promoters (Niu et al., 1996; Schultz et al., 1996).

Although it is possible that VP1 contacts the DNA, our data make it highly unlikely that VP1 binds by itself to the ABRE, which is in agreement with previous conclusions (McCarty et al., 1991). In vitro, VP1 interacts transiently and unspecifically with DNA (Hill et al., 1996). Proteins with these characteristics are difficult to detect by dimethyl sulfate (DMS) footprinting (Rigaud et al., 1991). Therefore, it cannot be excluded that VP1 binds to the rab28 promoter, although we have been unable to attribute a footprint to binding of VP1. Suzuki et al. (1997) showed that the B3 domain of VP1 binds specifically to the SPH element of the $\mathrm{C} 1$ promoter. The B3 domain is not necessary for activation through the ABRE (Vasil et al., 1995) and does not bind specifically to this element. Therefore, the results obtained for the interaction of VP1 with the SPH element do not account for activation through the ABRE (Suzuki et al., 1997).

The slight weakening of the footprint in the vp1 mutant points to VP1 as enhancing binding to the ABRE, as has been shown in vitro (Hill et al., 1996). However, the weakened footprint could be caused by vivipary and the desiccation deficiency of the mutant without being related to the mechanism of VP1 action via the ABRE.

It has been proposed that VP1 binds indirectly to the ABRE by protein-protein interactions with the ABF or an associated factor (Hattori et al., 1995; Vasil et al., 1995; Suzuki et al., 1997). In this scenario, VP1 functions as a coactivator that binds to a preformed promoter complex and stimulates transcription by means of its acidic activator domain (Hill et al., 1996). Induction by a coactivator would be expected to result in the same protein-DNA interactions in the wild-type and VP1-deficient embryos, in agreement with the in vivo footprinting presented here.

The different effects of VP1 on protein binding to the ABRE observed in vivo and in vitro must be due to the different binding conditions. In the in vitro experiment, the effect of VP1 was assayed on free DNA and protein (Hill et al., 1996). However, in vivo, there is near saturating binding to the ABRE in the absence of VP1. Proteins other than VP1 can enhance binding to the ABRE and could create a favorable environment for occupancy of the element in vivo (Schultz et al., 1996; P.K. Busk, M. Figueras, and M. Pagès, unpublished data). In excised embryos, especially without

from embryos of mature seeds incubated in $100 \mu \mathrm{M}$ ABA for the time indicated. All binding reactions contained $45 \mu \mathrm{g}$ of nuclear protein, except for 14-DAP embryos, for which $5 \mu \mathrm{g}$ of nuclear extract and $45 \mu \mathrm{g}$ of BSA were used. The retarded complex mentioned in the text is indicated by an arrow.

(B) The same as given in (A) but with $5 \mu \mathrm{g}$ of nuclear protein and 50 $\mu \mathrm{g}$ of BSA in each lane.

The $(-)$ indicates that no extract was added to the probe. 
$A B A$, the footprint on the ABREs became weaker. Thus, under these conditions, binding does not seem to be saturating and is therefore more like the experiment of Hill et al. (1996). Indeed, in excised embryos, the footprint on the ABREs was stronger in the wild type than it was in VP1deficient embryos, suggesting that VP1 can have a positive effect on binding to the ABRE in vivo. One caveat of this conclusion is that excised vp1 embryos have a germination capability different from that of the wild type (Robertson, 1955). The weaker footprints on the ABREs in excised VP1deficient embryos could therefore reflect the altered germination of the mutant rather than a direct effect of VP1 deficiency.

Although we cannot exclude that different proteins bind to ABRE in the presence and in the absence of VP1, as proposed by Vasil et al. (1995), our observations are not in agreement with this possibility. The similarity of the footprints on rab28 suggests that the same protein binds to the $A B R E$ in the wild type and the vp1 mutant. In addition, the same $A B F$ was present in extracts from both wild-type and vp1 embryos (P.K. Busk and M. Pagès, unpublished data). There could be a competition of different proteins with identical binding specificity on the ABRE. In this case, VP1 would specifically enhance binding of one of the two proteins to the ABRE. This is not in accordance with in vitro experiments in which VP1 enhances binding of all of the DNA binding proteins tested (Hill et al., 1996).

\section{Developmental Activation of rab28 through ABRE}

VP1 is necessary but not sufficient for developmental expression of rab28 because VP1 was detected in embryos as early as 10 DAP (McCarty et al., 1991), whereas rab28 mRNA was not detected before 20 DAP (Pla et al., 1991; Niogret et al., 1996). ABA is also involved in induction of rab28, but a comparison of rab28 expression in wild-type and $A B A-d e f i c i e n t$ embryos showed that there is no clear correlation between the $A B A$ level and the expression of rab28 (Pla et al., 1991). The current data suggest that a developmental factor (the postabscission factor) is the primary inducer of genes expressed during late embryogenesis (Hughes and Galau, 1991; Giraudat et al., 1994).

In our study, we found an ABF in nuclear extracts whose activity correlates with the expression of rab28. The factor disappeared in excised embryos but could be maintained by $A B A$ in the same way as the in vivo footprint on the ABREs of rab28. Therefore, it is a likely candidate for binding to the ABREs of rab28. This is supported by the fact that $A B F$ bound more strongly to ABRE $A$ than to ABRE $B$, which is in agreement with the relative strength of the in vivo footprints. These correlations suggest that the factor is relevant for the regulation of rab28 in vivo, but additional work is required to clarify this point.

The factor is likely to belong to the bZIP family of transcription factors, because the pattern of protection and hy- persensitivity on the two ABREs of the rab28 promoter in vivo resembles the footprint of the bZIP proteins that bind to ACGT-containing cis elements (Lu et al., 1996).

The correlation of the rab28 mRNA level with ABF activity but not with VP1 activity suggests that binding of ABF to the rab28 promoter is a prerequisite for stimulation of transcription by VP1. That transcription factors can regulate the activity of VP1-like proteins has been demonstrated in bean (Chern et al., 1996). However, expression of rab28 could also be regulated by a coactivator or a repressor that modifies the interaction of VP1 with the ABREs.

The disappearance of the ABF during germination suggests that it is embryo specific. It has been shown that ABRE A forms complexes of different mobility with nuclear proteins from embryos and leaves (Pla et al., 1993). The embryo-specific factor could be the maize homolog of the wheat protein EmBP-1, whose cDNA was cloned from an embryo cDNA library and binds to the ABRE (Guiltinan et al., 1990). The binding specificity of EmBP-1 (Niu and Guiltinan, 1994 ) is in agreement with a higher relative affinity for ABRE $A$ than for ABRE $B$.

\section{Regulation of rab28 by ABA}

Pla et al. (1993) showed that a fragment of 134 bp from the rab28 promoter is sufficient for induction by $A B A$ in rice protoplasts. Here, we have delineated four sequences in the same fragment that conferred $A B A$ inducibility upon a reporter gene in transient transformation in maize embryonic callus. In vivo footprinting of maize embryos confirmed the importance of the four elements investigated. The expression study should be interpreted carefully because of the transient character of the transformation data. However, the results seem to be relevant for regulation of rab28, but it cannot be excluded that sequences outside of the examined region are also important.

During precocious germination of excised embryos, the footprints became weaker but did not disappear, even though rab28 was not active (Niogret et al., 1996). In the vp1 mutant in which only low levels of rab28 mRNA are present (Pla et al., 1991), we observed the same footprints on the rab28 promoter as were found in the wild type. In addition, the footprints in the vp1 mutant did not change during induction with ABA. These results suggest that ABA and VP1 regulate rab28 by modification of a preformed promoter complex. The induction of rab28 by VP1 has been discussed above. ABA could stimulate rab28 transcription via induced binding of a cofactor or by post-translational modifications, such as changes in protein phosphorylation. Both protein phosphatases (Leung et al., 1994; Meyer et al., 1994) and kinases (Knetsch et al., 1996) affect ABA-induced gene expression, indicating that $A B A$ signal transduction involves phosphorylation and dephosphorylation events. Also, for the $A B A$-inducible gene rab17, there are footprints on the regulatory elements under uninduced conditions (Busk et al., 
1997). These results indicate that a modification of a preformed promoter complex is of major importance for ABAinduced transcription.

The two ABREs in the rab28 promoter are necessary for both ABA- and VP1-mediated induction in embryonic callus. Although the data from transient transformation should be interpreted with care, the results are in accordance with the clear footprints on the two ABREs in embryos. In addition, in vivo footprinting of the rab28 promoter in callus gave the same results (P.K. Busk and M. Pagès, unpublished data). In transient transformation, activation of the rab28 promoter by VP1 was stronger in the presence than in the abscence of $A B A$. This synergism between VP1 and ABA is typical of ABRE/G-box-mediated transcription (Hattori et al., 1992, 1995; Vasil et al., 1995) and mimicks the regulation in embryos. In embryos, the endogenous rab28 gene is less inducible by ABA in the vp1 mutant (Pla et al., 1991).

We are unable to explain the negative effect of VP1 on the activity of the ABRE $A^{-}$and $A B R E B^{-}$constructs. However, the effect was only seen without $A B A$. Under these conditions, the endogenous rab28 gene is not active in callus (M. $F$. Niogret and M. Pagès, unpublished data). The GRA element is an ABA- and water stress-inducible element from the rab17 promoter (Busk et al., 1997). In this study, we found a sequence with 7 of 12 bp identical to GRA, which is involved in the ABA response and had a weak footprint in embryos.

The coupling element CE3 is present in the promoter of the barley gene HVA1, where it is necessary for induction by ABA (Shen et al., 1996). In the rab28 promoter, the CE3-like element had a footprint in vivo, suggesting that it is involved in regulation of rab28. This is further supported by the importance of the element for induction by $A B A$ in transient transformation. Shen et al. (1996) showed that CE3 together with an ABRE confer ABA inducibility upon a minimal promoter, whereas none of the elements functions alone. CE1 and an ABRE from HVA22 also function together but cannot function alone (Shen and Ho, 1995). These results together with our finding that a combination of two ABREs in front of a $35 S$ promoter was $A B A$ inducible suggest that a combination of the ABRE with any other ABA-inducible element is sufficient to determine ABA response. Puente et al. (1996) described a similar regulation controlling light-responsive transcription.

\section{METHODS}

\section{In Vivo Footprinting and DNA Purification}

Wild-type embryos (Zea mays) of the pure inbred line W64A and homozygous viviparous1 (vp1) mutants (Pla et al., 1991) were used for in vivo footprinting with dimethyl sulfate (DMS) within 15 min after excision from the cob or after $24 \mathrm{hr}$ of incubation with $100 \mu \mathrm{M}$ abscisic acid (ABA) or with water (Busk et al., 1997). The DNA was purified and cleaved at DMS-modified guanines (Busk et al., 1997).

\section{Ligation-Mediated Polymerase Chain Reaction}

Ligation-mediated polymerase chain reaction (LMPCR) was performed as described previously (Busk et al., 1997). Two millimolar $\mathrm{MgSO}_{4}$ was used in all PCR steps.

The sequence of oligonucleotides for LMPCR of the transcribed strand in the wild-type W64A is (in the order of first primer, second primer, and third primer) h1, 5'-CTACTACAGTACAGATCACGGCTTG-3'; h2, 5'-AGCTGCCTCTTGAGACTTGATGGTG-3'; and h3, 5'-GCCTCTTGAGACTTGATGGTGAGTGTGG-3'. Another primer set was used for the $v p 1$ mutant due to differences in the sequence of the promoter and is as follows: $h 3$ and $h 4,5^{\prime}$-GGATCGTTGCGATTATATGCGGCGT-3'; and h5, 5'-TTGCGATTATATGCGGCGT(A/G)CGGGGAG- $3^{\prime}$. For the nontranscribed strand, the oligonucleotides were i1, 5'-ACACGCATGCCCTGATGCTC-3'; 2 , 5'-CGGGAGCTTCTTCATCCAGCTTG-3'; and i3, 5'-CGGGAGCTTCTTCATCCAGCTTGCAG-3'.

\section{Autoradiography and Scanning}

AGFA Curix (AGFA, Leverkusen, Germany) or X-AR (Kodak, Rochester, NY films were exposed at $-70^{\circ} \mathrm{C}$ with an intensifying screen for 10 $\mathrm{hr}$ to 7 days.

All footprints were confirmed by scanning. For each experiment, several independent amplifications were performed by using LMPCR. No variation in band intensity was observed between amplifications. For quantitative evaluation, the films were scanned and the intensity of each peak was determined (Busk et al., 1997).

\section{Plasmid Constructs}

The wild-type promoter- $\beta$-glucuronidase (GUS) fusion construct was pMP130, which contains the rab28 sequence from positions -393 to -60 fused to the cauliflower mosaic virus $35 \mathrm{~S}-90$ to +8 promoter in front of GUS and nosT (Pla et al., 1993).

Mutants were constructed by recombinant PCR as described previously (Cormack, 1992). The following mutants were obtained (sequences from $5^{\prime}$ to $3^{\prime}$, with wild-type bases in uppercase letters and mutated bases in lowercase letters and the numbering relative to the transcription start point): ABRE $A^{-},(-152)$ CGCGCCtgcaGGGCATG (-135); ABRE $B^{-},(-110)$ TCGTCTgaAttcCTCTC (-94); GRA ${ }^{-},(-142)$ TGGGCActCgagCGCGA $(-126)$; and CE3 ${ }^{-},(-128)$ CGACtCtagaCagCTTG (-112). The ABRE $A^{-} B^{-}$mutant contained the ABRE $A^{-}$ and $A B R E B^{-}$mutations.

The plasmid with ABRE $A$ and $A B R E B$ in front of a $-9035 S$ promoter was constructed by annealing the oligonucleotides (sequences from $5^{\prime}$ to $3^{\prime}$, with rab28 sequences in uppercase letters and other bases in lowercase letters) agcttGCCACGTGGGagatctgtcgacTCCACGTCTCc and tcgagGAGACGTGGAgtcgacagatcaCCCACGTGGCa. The double-stranded oligonucleotide was inserted in pMP130, which had been digested with Hindlll and Xhol. The -90 35S promoter construct was made by cutting out the rab28 promoter of pMP130 with Sall and Xhol and closing the vector fragment. All constructs were confirmed by DNA sequencing. 35S-Sh-Vp1 expresses VP1 under the control of a double $35 S$ promoter and with an intron from the shrunken gene to increase expression. This plasmid is described by McCarty et al. (1991). The plasmid pRT-ex/s-int/s-LUC (Maas et al., 1991) was used as internal control in transformation assays. 


\section{Transient Transformation}

Callus was made from the maize line Black Mexican Sweet and maintained as described by Vilardell et al. (1990) with $3 \mathrm{mg} / \mathrm{mL} 2,4-$ dichlorophenoxyacetic acid in the medium. Approximately $1 \mathrm{~g}$ of callus was spread on filter paper 1 week after replication and incubated overnight at $26^{\circ} \mathrm{C}$ in the dark. Four hours before bombardment, the callus was moved to medium with $200 \mathrm{mM}$ mannitol. The callus was transformed by particle bombardment with $4 \mu \mathrm{g}$ of the rab28-GUS plasmid and $2 \mu \mathrm{g}$ of pRT-ex/s-int/s-LUC as an internal standard or with $3 \mu \mathrm{g}$ of rab28-GUS plasmid DNA, $1.5 \mu \mathrm{g}$ of $35 \mathrm{~S}-\mathrm{Sh}-\mathrm{Vp} 1$, and $1.5 \mu \mathrm{g}$ of $\mathrm{pRT}$-ex/s-int/s-LUC, essentially as described by Klein et al. (1987).

Each sample was divided in two after transformation and incubated in medium with or without $100 \mu \mathrm{M} \mathrm{ABA}$ for $22 \mathrm{hr}$ in the dark at $26^{\circ} \mathrm{C}$ before freezing in liquid nitrogen. Luciferase and GUS assays were done as previously described (Busk et al., 1997). Relative GUS activity is the reading of the GUS assay divided by the reading of the luciferase assay.

\section{Nuclear Extracts}

Nuclear extracts were prepared from embryos, as described by Busk and Pagès (1997)

\section{Electrophoretic Mobility Shift Assays}

The following DNA oligonucleotides were used for electrophoretic mobility shift assays (symbols are as given above): ABRE A, $5^{\prime}$-tggaagcttCGCGCCACGTGGGCATGagatct- $3^{\prime}$ and $5^{\prime}$-tggagatctCATGCCCACGTGGCGCGaagctt-3'; ABRE A-, 5' -tggaagcttCGCGCCtgcaGGGCATGagatct-3' and 5'-tggagatctCATGCCCtgcaGGCGCGaagctt3'; ABRE B, 5' -tggaagcttGTCTCCACGTCTCTCGCagatct-3' and 5' -tggagatctGCGAGAGACGTGGAGACaagctt- $3^{\prime}$; and ABRE $B^{-}, 5^{\prime}$-tggaagcttGTCTgaAttcCTCTCGCagatct-3' and 5'-tggagatctGCGAGAGgaaTtcAGACaagctt-3'.

The full-length oligonucleotides were purified by denaturing PAGE. Complementary oligonucleotides were annealed and purified in a nondenaturing polyacrylamide gel.

The double-stranded oligonucleotides were labeled with $\alpha^{-32} \mathrm{P}$-dATP $(3000 \mathrm{Ci} / \mathrm{mmol}$, Amersham) by filling in with the Klenow fragment of DNA polymerase I (Sambrook et al., 1989) and purified on a NAP5 column (Pharmacia), according to the manufacturer's instructions. Unlabeled competitor DNA was filled in with nonradioactive nucleotides.

The radioactive probe $(10,000 \mathrm{cpm})$ was incubated with 5 to $45 \mu \mathrm{g}$ of nuclear extract in $10 \mu \mathrm{L}$ of $1 \times$ binding buffer $(25 \mathrm{mM}$ Hepes, $\mathrm{pH}$ $7.8,75 \mathrm{mM} \mathrm{KCl}, 5 \mathrm{mM} \mathrm{MgCl}_{2}, 0.5 \mathrm{mM}$ EDTA, $0.2 \mathrm{mM} \mathrm{DTT}$, and $10 \%$ glycerol) and $150 \mathrm{ng}$ of poly(dl-dC) for $20 \mathrm{~min}$ on ice before loading on a $1 \times$ Tris-borate-EDTA, $5 \%$ (30:0.8 acrylamide-bis) polyacrylamide gel. Electrophoresis was at $10 \mathrm{~V} / \mathrm{cm}$ at $4^{\circ} \mathrm{C}$. In competition experiments, the nuclear extract was preincubated with the competitor in $8 \mu \mathrm{L}$ of $1 \times$ binding buffer and $120 \mathrm{ng}$ of poly(dl-dC) for $10 \mathrm{~min}$ on ice before the radioactive probe was added.

\section{ACKNOWLEDGMENTS}

We thank Dr. Don McCarty for the gift of the VP1 expression plasmid. We thank Pilar Fontanet for help with growth and maintenance of maize plants and cell culture, Alison Jessop for advice on callus transformation, and Dr. Niels A. Lassen for comments on the manuscript. This work was funded by the European Community BIOTECH Program, Grant No. B104-C796-0062, and by Grant No. BIO-971211 from Plan Nacional de Investigación Científica y Desarrollo Tecnológico. P.K.B. was supported by a fellowship from the Danish Natural Science Research Council.

Received May 12, 1997; accepted October 27, 1997.

\section{REFERENCES}

Busk, P.K., and Pagès, M. (1997). Microextraction of nuclear proteins for EMSA from single maize embryos. Plant Mol. Biol. Rep., in press.

Busk, P.K., Jensen, A.B., and Pagès, M. (1997). Regulatory elements in vivo in the promoter of the abscisic acid responsive gene rab17 from maize. Plant J. 11, 1285- 2295.

Chern, M.-S., Bobb, A.J., and Bustos, M.M. (1996). The regulator of MAT2 (ROM2) protein binds to early maturation promoters and represses PvALF-activated transcription. Plant Cell 8, 305-321.

Cormack, B. (1992). Mutagenesis by the polymerase chain reaction. In Current Protocols in Molecular Biology, F.M. Ausubel, R. Brent, R.E. Kingston, D.D. Moore, J.G. Seidman, J.A. Smith, and K. Struhl, eds (New York: Wiley Interscience), pp. 8.5.1-8.5.4 (suppl. 15).

Giraudat, J., Parcy, F., Bertauche, N., Gosti, F., Leung, J., Morris, P.C., Bouvier-Durand, M., and Vartanian, N. (1994). Current advances in abscisic acid action and signalling. Plant Mol. Biol. 26, 1557-1577.

Giuliano, G., Pichersky, E., Malik, V.S., Timko, M.P., Scolnik, P., and Cashmore, A.R. (1988). An evolutionarily conserved protein binding sequence upstream of a plant light-regulated gene. Proc. Natl. Acad. Sci. USA 85, 7089-7093.

Guiltinan, M.J., Marcotte, W.R., and Quatrano, R.S. (1990). A plant leucine zipper protein that recognizes an abscisic acid response element. Science 250, 267-271.

Hattori, T., Vasil, V., Rosenkrans, L., Hannah, L.C., McCarty, D.R., and Vasil, I.K. (1992). The viviparous-1 gene and abscisic acid activate the $C 1$ regulatory gene for anthocyanin biosynthesis during seed maturation in maize. Genes Dev. 6, 609-618.

Hattori, T., Terada, T., and Hamasuna, S. (1995). Regulation of the Osem gene by abscisic acid and the transcriptional activator VP1: Analysis of cis-acting promoter elements required for regulation by abscisic acid and VP1. Plant J. 7, 913-925.

Hill, A., Nantel, A., Rock, C.D., and Quatrano, R.S. (1996). A conserved domain of the viviparous-1 gene product enhances the DNA binding activity of the bZIP protein EmBP-1 and other transcription factors. J. Biol. Chem. 271, 3366-3374.

Hughes, D.W., and Galau, G.A. (1991). Developmental and environmental induction of Lea and LeaA mRNAs and the postabscission program during embryo culture. Plant Cell 3, 605-618.

Ingram, J., and Bartels, D. (1996). Molecular basis of dehydration tolerance in plants. Annu. Rev. Plant Physiol. Plant Mol. Biol. 47, $377-403$. 
Izawa, T., Foster, R., and Chua, N.-H. (1993). Plant bZIP protein DNA binding specificity. J. Mol. Biol. 230, 113t-1144.

Klein, T.M., Wolf, E.D., Wu, R., and Sanford, J.C. (1987). Highvelocity microprojectiles for delivering nucleic acids into living cells. Nature 327, 70-73.

Knetsch, M.L.W., Wang, M., Snaar-Jagalska, B.E., and Heimovaara-Dijkstra, S. (1996). Abscisic acid induces mitogenactivated protein kinase activation in barley aleurone protoplasts. Plant Cell 8, 1061-1067

Leung, J., Bouvier-Durand, M., Morris, P.C., Guerrier, D., Chefdor, F., and Giraudat, J. (1994). Arabidopsis ABA response gene $A B$ /1: Features of a calcium-modulated protein phosphatase. Science 264, 1448-1452

Lu, G., Paul, A.-L., McCarty, D.R., and Ferl, R.J. (1996). Transcription factor veracity: Is GBF3 responsible for ABA-regulated expression of Arabidopsis Adh? Plant Cell 8, 847-857.

Maas, C., Laufs, J., Grant, S., Korfhage, C., and Werr, W. (1991). The combination of a novel stimulatory element in the first exon of the maize Shrunken-1 gene with the following intron 1 enhances reporter gene expression up to 1000-fold. Plant Mol. Biol. 16, 199-207.

Marcotte, W.D., Jr., Russell, S.H., and Quatrano, R.S. (1989). Abscisic acid-responsive sequences from the $\mathrm{Em}$ gene of wheat. Plant Cell 1, 969-976.

McCarty, D.R. (1995). Genetic control and integration of maturation and germination pathways in seed development. Annu. Rev. Plant Physiol. Plant Mol. Biol. 46, 71-93.

McCarty, D.R., Hattori, T., Carson, C.B., Vasil, V., Lazar, M., and Vasil, I.K. (1991). The Viviparous-1 developmental gene of maize encodes a novel transcriptional activator. Cell 66, 895-905.

McKendree, W.L., Jr., and Ferl, R.J. (1992). Functional elements of the Arabidopsis Adh promoter include the G-box. Plant Mol. Biol. 19, 859-862.

Meyer, K., Leube, M.P., and Grill, E. (1994). A protein phosphatase 2C involved in $\mathrm{ABA}$ signal transduction in Arabidopsis thaliana. Science 264, 1452-1455.

Mundy, J., Yamaguchi-Shinozaki, K., and Chua, N.-H. (1990). Nuclear proteins bind conserved elements in the abscisic acidresponsive promoter of a rice $R A B$ gene. Proc. Natl. Acad. Sci. USA $87,1406-1410$

Niogret, M.F., Culiáñez-Macià, F.A., Goday, A., Albà, M.M., and Pagès, M. (1996). Expression and cellular localization of rab28 mRNA and Rab28 protein during maize embryogenesis. Plant $\mathrm{J}$. 9, 549-557.

Niu, X., and Guiltinan, M.J. (1994). DNA binding specificity of the wheat bZIP protein EmBP-1. Nucleic Acids Res. 22, 4969-4978.

Niu, X., Adams, C.C., Workman, J.L., and Guiltinan, M.J. (1996). Binding of the wheat basic leucine zipper protein EmBP-1 to nucleosomal binding sites is modulated by nucleosome positioning. Plant Cell 8, 1569-1587.

Parcy, F., Valon, C., Raynal, M., Gaubier-Comella, P., Delseny, M., and Giraudat, J. (1994). Regulation of gene expression programs during Arabidopsis seed development: Roles of the $A B / 3$ locus and of endogenous abscisic acid. Plant Cell 6, 1567-1582.
Pla, M., Gómez, J., Goday, A., and Pagès, M. (1991). Regulation of the abscisic acid-responsive gene rab28 in maize viviparous mutants. Mol. Gen. Genet. 230, 394-400.

Pla, M., Vilardell, J., Guiltinan, M.J., Marcotte, W.R., Niogret, M.F., Quatrano, R.S., and Pagès, M. (1993). The cis-regulatory element CCACGTGG is involved in $A B A$ and water-stress responses of the maize gene rab28. Plant Mol. Biol. 21, 259-266.

Puente, P., Wei, N., and Deng, X.W. (1996). Combinatorial interplay of promoter elements constitutes the minimal determinants for light and developmental control of gene expression in Arabidopsis. EMBO J. 15, 3732-3743.

Rigaud, G., Roux, J., and Pictet, R. (1991). In vivo footprinting of rat TAT gene: Dynamic interplay between the glucocorticoid receptor and a liver-specific factor. Cell 67, 977-986.

Robertson, D.S. (1955). The genetics of vivipary in maize. Genetics 40, 745-760.

Salinas, J., Oeda, K., and Chua, N.-H. (1992). Two G-box-related sequences confer different expression patterns in transgenic tobacco. Plant Cell 4, 1485-1493.

Sambrook, J., Fritsch, E.F., and Maniatis, T. (1989). Molecular Cloning: A Laboratory Manual. (Cold Spring Harbor, NY: Cold Spring Harbor Laboratory Press).

Schultz, T.F., Spiker, S., and Quatrano, R.S. (1996). Histone H1 enhances the DNA binding activity of the transcription factor EmBP-1. J. Biol. Chem. 271, 25742-25745.

Shen, Q., and Ho, T.-H.D. (1995). Functional dissection of an abscisic acid (ABA)-inducible gene reveals two independent ABA-responsive complexes each containing a G-box and a novel cis-acting element. Plant Cell 7, 295-307.

Shen, Q., Zhang, P., and Ho, T.-H.D. (1996). Modular nature of abscisic acid (ABA) response complexes: Composite promoter units that are necessary and sufficient for ABA induction of gene expression in barley. Plant Cell 8, 1107-1119.

Suzuki, M., Kao, C.Y., and McCarty, D.R. (1997). The conserved B3 domain of VIVIPAROUS1 has a cooperative DNA binding activity. Plant Cell 9, 799-807.

Vasil, V., Marcotte, W.R., Jr., Rosenkrans, L., Cocciolone, S.M., Vasil, I.K., Quatrano, R.S., and McCarty, D.R. (1995). Overlap of Viviparous1 (VP1) and abscisic acid response elements in the Em promoter: G-box elements are sufficient but not necessary for VP1 transactivation. Plant Cell 7, 1511-1518.

Vilardell, J., Goday, A., Freire, M.A., Torrent, M., Martínez, M.C., Torné, J.M., and Pagès, M. (1990). Gene sequence, developmental expression, and protein phosphorylation of RAB-17 in maize. Plant Mol. Biol. 14, 423-432.

Weisshaar, B., Armstrong, G.A., Block, A., Da Costa e Silva, O., and Hahlbrock, K. (1991). Light-inducible and constitutively expressed DNA-binding proteins recognizing a plant promoter element with functional relevance in light responsiveness. EMBO J. 10, 1777-1786.

Williamson, J.D., and Scandalios, J.G. (1992). Differential response of maize catalases to abscisic acid: Vp1 transcriptional activator is not required for abscisic acid-regulated Cat1 expression. Proc. Natl. Acad. Sci. USA 89, 8842-8846. 